\title{
Editorial: The humanity of engagement at the core of developing and sharing knowledge
}

\author{
Sophie Duncan* - National Co-ordinating Centre for Public Engagement, UK \\ Sandy Oliver* - UCL Institute of Education, UK
}

Research for All has come a long way in the three years since we began working on the first issue. We have better understandings of: how to work with a team of associate editors from around the world; the multidisciplinary and multi-practice approaches of engaged scholarship; the need to support authors and peer reviewers to work collaboratively to improve the quality of all our work; the importance of encouraging collaborators to co-write; and the challenges in seeking pieces from across different cultures, contexts and languages.

Learning together has been a central facet of how we have developed the journal. We are constantly adapting and refining our approach as we draw on the traditions, insights and expertises of a diverse group of people committed to engagement, not as an end in itself, but as a core part of developing and sharing knowledge.

From the beginning, we have sought to live out engaged practice in how we develop the journal. Engagement is a human process, requiring the ability to listen, to be curious, to learn and change, to be challenged, and to grow. It requires empathy, understanding, patience, tenacity and humility, and an ability to recognize our biases, our limitations and our collective potential. The humanity of these processes is both enticing and challenging.

In a recent report, Lowe and Plimmer (2019) reflect on the need for what the authors describe as a human, learning, systemic approach (HSL) to achieving social outcomes. Rather than focus on the desired outcomes, an HSL approach focuses on the centrality of people to effect change. The report suggests that, to respond effectively to the complexity of the world, funders, commissioners and those who work on the ground need to encourage:

- Being human to one another: they recognize and respond to human variety with bespoke support; they build empathy between people; they recognize the strengths of others; and they seek to trust and be trusted.

- Using learning to enable performance improvement: they use a variety of both quantitative and qualitative data to learn; they create learning cultures; and they fund and commission for learning, not for the delivery of specified services.

- Looking after the health of the systems which create social outcomes: they create the conditions in which people can understand the systems of which they are part, and enable effective collaboration and coordination of actors within these systems. (Collaborate CIC, 2019)

These three aspects are so pertinent to the role of engaged research, where human relationships are essential to effective work; where learning is embedded as a key part of the process, enabling adaptation and change, leading to improved processes and outcomes for all; and where a recognition of the context and systems helps inform the roles people can play in effecting change. 
Therefore, it is not a surprise that the principles of HSL are reflected in the contributions to this issue. Wihofszky and Sternberg evidence the value of people-centric connections in developing a 'health-promoting social culture in the neighbourhoods and municipalities' (201). By connecting participatory research transnationally, participants were able to learn from one another, and adopted more health-friendly behaviours. The authors reflect on how these human processes need time, a point reinforced by Wilkins and Cooper, who share ten lessons they have learned about knowledge exchange while cultivating a knowledge-exchange network in the UK. In one lesson, they describe the importance of building trust, credibility and openness, stating that 'resources need to be made available to support the time and energy that need to be invested, first in gaining and then in maintaining such trust' (212). Their lessons exemplify an HLS approach - supporting the need for learning and adaptation, and highlighting the need to create the conditions where knowledge exchange can flourish, including: 'creating a safe, shared space to enable opportunities for engagement and the building of trust between researchers, policy and practitioners; translating and communicating science for practical decision making; understanding of the varied and changing needs of researchers and stakeholders; and knowing how to manage these ingredients into a successful mix' (215).

At a Research for All seminar in May 2019, Rick Holliman, Professor of Engaged Research at the Open University, and a member of the Research for All Advisory Board, talked about the moral imperatives that underpin engagement. He referenced Fabien Medvecky's (2018) concept of 'fairness in knowing', the idea that the current ways the world works marginalize people from benefiting from knowledge, and contributing to its creation. This driver has seen his research career draw on traditions of participatory research, ensuring that engagement is a key facet of what it means to be a researcher, as referenced in his recent inaugural lecture (Holliman, 2019).

The need for fairness in knowing has also caused us to reflect on the language of the journal - and consider what we would need to do to enable contributions in different languages, and different formats. Over time, we hope contributors will be inspired to share their poetry, films, artwork and stories, and that we will find effective ways to involve contributions in people's first language when this is not English. To do this, we will need to find a wider range of peer reviewers from across the world, and more significant resourcing, but it is something we aspire to do.

While for many, 'fairness in knowing' motivates their approach to participatory research, it is not without its challenges, as picked up by Lenette et al. As a group of women researchers who recognize participatory research as an important part of the academy, the authors are keen to examine the emotional and ethical challenges involved in doing it well. The piece considers the gendered aspects of participatory research, and reflects on debates about what counts as legitimate or important academic practice and knowledge. They talk about the emotional load of these ways of working, and the lack of visibility and criticality in reflecting on this. They consider the challenges of creating trustful relationships, and the potential blurred boundaries of friendship and professional relationships cultivated in this work. They reflect that 'virtues of care, compassion and equality upheld by many academic researchers engaged in participatory research can also become their "burdens", sometimes even putting them in "dangerous situations" where they can get hurt' (174). In addition, they highlight the conflict between creating non-hierarchical relationships in developing the research, and the fact that many cultures have implicit hierarchies that need to be respected. They suggest that there is a 'need to make sense of how "democracy" 
could be understood and negotiated in the everyday life practices of people ... which may emphasize "hierarchical harmony" over "non-hierarchical dialogue"' (167).

While many are motivated by a desire for fairness in knowing, others are motivated to engage the public with research for other reasons. Understanding these motivations is a key to developing effective interventions, as illustrated in our current issue. Motivated by a desire to see patients benefit from research, Gilbert et al. worked with patients with dry age-related macular degeneration (AMD) to explore their challenges to taking on research findings in their lives. Working with a team of people from inside and outside of the university, they organized a 'participantinformed community kitchen programme', which drew on a range of engagement methodologies to encourage people to make changes to their diet that could be beneficial to their eye health.

Sometimes the motivations can undermine the engagement work, and lead to challenges. Rempel et al. consider public engagement in government, highlighting the implicit assumptions behind public engagement in data science ethics. They point out that the multiplicity of engagement purposes undermined the quality of the work undertaken. A further challenge related to assumptions that organizers made about publics. By imagining a public who are neither strongly supportive of nor against data science, they illustrate how the choice of participants supported this assumption, which meant that it was not possible to truly assess public views of the topic. Their case study is an example that illustrates how important it is to understand the system you are part of, and the various actors within it.

Understanding publics can lead to really effective means to engage with them. Hobbs et al. tapped into their potential participants' interest in the game Minecraft to encourage young people to engage with science. Testing this approach in a variety of settings, including at science festivals and non-science-related events, they were able to confirm that, for many participants, Minecraft was a big enough draw to encourage them to engage with science.

Williams is concerned with how to evidence social change by considering the system within which such changes might occur. Reflecting on impacts of broadcast, he directs our attention to the relationships academics develop with media professionals, reminding us that 'networking is fundamental to many forms of impact' (221). He goes on to argue that the relationships between academics and media professionals can shape both broadcast and research, and that academics who are successful in broadcast are those who are able to speak the language of a 'world beyond their own' (222).

All our contributors are reflecting on the humanity of engagement. As researchers, participants, collaborators and practitioners who come together because they care about high-quality research that draws on a range of perspectives and insights and contributes to improved social outcomes, they recognize that human relationships sit at the core. The HSL approach helps us to remember that the very processes of engagement, as reflected in the pages of the journal, matter, and that by understanding how to learn together, recognizing the complexity of the world in which we operate, and by adapting and changing, we can improve what we do, and by valuing the time taken to cultivate relationships, we can contribute to a better world.

\section{Articles in this issue}

Gilbert, R., Rawlings, A., Dixon, M., Gonçalves de Pinho, A.R. and Caffrey, T. (2019) 'Eating for Eye Health: Engaging patients with dry age-related macular degeneration in community cookery to support lifestyle change and positive health', Research for All, 3 (2), 129-41. 
Hobbs, L., Stevens, C., Hartley, J., Ashby, M., Lea, I., Bowden, L., Bibby, J., Jackson, B., McLaughlin, R. and Burke, T. (2019) 'Using Minecraft to engage children with science at public events', Research for All, 3 (2), 142-60.

Lenette, C., Banks, S., Nunn, C., Coddington, K., Cook, T., Kong, S.T. and Stavropoulou, N., (2019) 'Brushed under the carpet: Examining the complexities of participatory research', Research for All, 3 (2), 161-79.

Rempel, E.S., Barnett, J. and Durrant, H. (2019) 'The hidden assumptions in public engagement: A case study of engaging on ethics in government data analysis', Research for All, 3 (2), 180-90

Wihofszky, P. and Sternberg, A. (2019) 'Promoting health through personal change in social networks: A German-Danish partnership', Research for All, 3 (2), 191-203.

Wilkins, T. and Cooper, I. (2019) 'Lessons from coordinating a knowledge-exchange network for connecting research, policy and practice', Research for All, 3 (2), 204-17.

Williams, S.M. (2019) 'The research impact of broadcast programming reconsidered: Academic involvement in programme-making', Research for All, 3 (2), 218-23.

\section{Acknowledgements}

Research for All is a collaborative project that relies on a range of associate editors with experience of engaged research in a variety of contexts. The associate editors who advised us on the content for this issue of the journal are:

- Cath Chamberlain - Baker IDI Heart and Diabetes Institute, Australia

- Trevor Collins - Open University, UK

- Helen Featherstone - University of Bath, UK

- Jamie Gallagher - University of Glasgow, UK

- Ceri Davies - University of Brighton, UK

- Henk Mulder - Groningen University, The Netherlands

- Gene Rowe-Gene Rowe Associates, UK

- Clare Wilkinson - University of the West of England, UK

We extend our thanks to them and to all the expert reviewers - two for each contribution - who helped us guide authors towards their final drafts.

\section{References}

Collaborate CIC (2019) 'Exploring the new world: practical insights for funding, commissioning and managing in complexity', blog post, 19 March. Online. https://collaboratecic.com/exploringthe-new-world-practical-insights-for-funding-commissioning-and-managing-incomplexity20a0c53b89aa (accessed 1 August 2019).

Holliman, R. (2019) 'Fairness in knowing: How should we engage with the sciences?'. Inaugural lecture, Open University, 12 March 2019. Online. https://tinyurl.com/y6lhwvew (accessed 25 June 2019).

Lowe, T. and Plimmer, D. (2019) Exploring the New World: Practical insights for funding, commissioning and managing in complexity. London: Collaborate. Online. https://tinyurl.com/ y53wzexh (accessed 25 June 2019).

Medvecky, F. (2018) 'Fairness in knowing: Science communication and epistemic justice'. Science and Engineering Ethics, 24 (5), 1393-408. 